\title{
Valores ambientales y su efecto mediador entre características demográficas y resultados empresariales ${ }^{*}$
}

\author{
Sánchez-Medina, Patricia S.* \\ Toledo-López, Arcelia** \\ Bautista-Cruz, Angélica** \\ Regino-Maldonado, Juan ${ }^{\star *}$
}

\section{Resumen}

El objetivo de este artículo es analizar el efecto mediador de los valores ambientales en la relación entre las características demográficas (sexo, escolaridad, edad y etnia) y los resultados empresariales (desempeño económico y desempeño ambiental), bajo la perspectiva de procesos intervinientes de la demografía organizacional. Para cumplir con este objetivo, se llevó a cabo una investigación cuantitativa de diseño transversal, con base en un análisis factorial y de regresión lineal múltiple, sobre una muestra constituida por 168 artesanos, dueños de negocios de alfarería de los estados de Oaxaca, Puebla y Tlaxcala, en México. Los resultados muestran un efecto mediador total de los valores ambientales en la relación entre el sexo y el desempeño empresarial, así como un efecto mediador parcial en la relación entre escolaridad y desempeño empresarial; no se encontró efecto mediador alguno de los valores ambientales en la relación entre edad y desempeño empresarial ni en la relación entre etnia y desempeño empresarial.

Palabras clave: Características demográficas, valores ambientales, desempeño económico, desempeño ambiental, negocios de artesanías.

\section{Recibido: 25-09-12 Aceptado: 22-05-14}

* Está investigación fue financiada por el proyecto: Características socio-demográficas, valores y el desempeño económico y ambiental de los negocios de artesanías de barro en México. Clave: SIP 20110528, del Instituto Politécnico Nacional.

** Profesores- Investigadores del CIIDIR, Instituto Politécnico Nacional, Unidad Oaxaca, México E-mail: psanchez@ipn.mx, E-mail: arcetole@hotmail.com, E-mail: angeli73@hotmail.com, E-mail: juanregino@hotmai.com 


\title{
Environmental Values and their Mediating Effect on the Relationship between Demographic Characteristics and Business Results
}

\begin{abstract}
The aim of this paper is to examine the mediating effect of environmental values on the relationship between demographic characteristics (sex, education, age and ethnicity) and a company's results (economic performance and environmental performance), from the perspective of the processes involved in organizational demography. A quantitative study with a cross-sectional design was carried out, based on factor analysis and multiple linear regressions, using a sample of 168 pottery business owners from the states of Oaxaca, Puebla and Tlaxcala, Mexico. The results show a total mediating effect of environmental values on the relationship between gender and business performance, as well as a partial mediating effect on the relation between schooling and business performance. No mediating effect was found for environmental values on the relationship between age and company performance or on the relationship between ethnicity and business performance.
\end{abstract}

Key words: Demographic characteristics, environmental values, economic performance, environmental per formance, handicraft businesses.

\section{Introducción}

La teoría demográfica ha generado desde tiempo atrás considerable interés debido a su importancia teórica en el estudio de las organizaciones (Stinchcombe et al., 1968); no obstante, dicha teoría genera desconcierto, ya que explicaciones demográficas simples pueden generar frecuentemente diversas posturas $\mathrm{y} / \mathrm{o}$ enfoques, los cuales involucran explicaciones diversas y numerosos conceptos adicionales, llevando el tema de la demografía organizacional a un ámbito complejo de interpretar.

De acuerdo con Pfeffer (1983), la literatura sobre demografía organizacional presenta la dificultad de uniformar conceptos y problemas en su interpretación teórica. Según este autor, estos problemas se deben a conceptos tales como: actitudes, cogniciones y valores, los cuales no pueden ser medidos directamente. Así, la idea central de Pfeffer (1983) se basa en plantear que las características o variables demográficas de los individuos, como el sexo, escolaridad, etnia y edad, son superiores que sus características psicológicas ${ }^{1}$, como valores, actitudes y cogniciones, para entender los resultados de la organización, por dos razones principales: 1) las características demográficas son directamente observables $y$, por ende, son más confiables y sus medi- 
das son validadas, y 2) por sustituir características psicológicas, las características demográficas producen modelos del comportamiento humano más rigurosos. Estas aseveraciones han originado lo que Lawrence (1997) llamó la "caja negra" de la demografía organizacional, porque no se sabe qué sucede con las características psicológicas, también conocidas como variables subjetivas, y sus relaciones dentro de los modelos de investigación. Así, el principal problema que se identifica dentro de los estudios demográficos es que no se prueban las variables subjetivas, lo cual corre el riesgo de un vago o falso análisis en la interpretación de los efectos de las variables demográficas en los resultados de la organización (Haas, 2010; Jackson et al., 1991; Khan y Vieito, 2013; Parrota et al., 2014; Robb y Watson, 2012; Wiersema y Bantel, 1993).

Asimismo, las investigaciones que se enfocan en explicar la "caja negra" de la demografía organizacional tienden a ignorar los valores. La mayoría de estos estudios se han centrado en analizar el papel del conflicto como variable interviniente entre la diversidad demográfica y los resultados de la empresa (De Wit y Greer, 2008: Kearney et al., 2009; Sobral y Bisseling, 2012).

Contrario a los argumentos de Pfeffer (1983), el objetivo de este artículo es analizar el efecto mediador de los valores ambientales $^{2}$ en la relación entre las ca- racterísticas demográficas y los resultados empresariales, considerando el hecho de que las características demográficas por sí mismas no pueden ser indicadores fiables de variables subjetivas. Además, se pretende contribuir teóricamente a la literatura sobre demografía organizacional, en donde no se han abordado temas sobre aspectos ambientales, como se propone en esta investigación. Se propone un modelo en el cual los valores ambientales, como variable subjetiva, explican la relación entre características demográficas, como el sexo, escolaridad, etnia y edad, y los resultados empresariales (desempeño económico y ambiental) en negocios de artesanías de alfarería, en donde las características demográficas de los propietarios son factores importantes que influyen para lograr una mejor competitividad (Domínguez et al., 2004); por tanto, estas características podrían ser elementos sustanciales para explicar diferencias respecto a los resultados de los negocios de artesanías. Los valores, por otra parte, no solo son importantes dentro de la literatura de demografía organizacional para explicar con mayor detalle el vínculo entre características demográficas y resultados de la empresa, sino también son una variable trascendental para los artesanos, ya que estos tienen ideas muy tradicionalistas, arraigadas en sus creencias y valores.

En México, se estima que la población dedicada de tiempo completo a la acticuidado del ambiente natural. Para los fines de este estudio, son pautas que enfatizan una relación armoniosa entre el artesano y la naturaleza. 
vidad artesanal es de 507,368 artesanos (CESOP, 2012). Para los fines de esta investigación, el universo se define como el conjunto de artesanos dedicados de tiempo completo a la actividad artesanal y que radican en México, en los estados de Oaxaca (58,398 artesanos), Puebla $(28,354)$ o Tlaxcala $(10,157)$, haciendo un total de 96,909 artesanos. El muestreo se realizó por conveniencia, seleccionando aquellos artesanos dueños de negocios de alfarería. Además, se aplicaron los siguientes criterios: viabilidad de la logística, accesibilidad en costos y representatividad de los estados. Considerando estos criterios, así como un nivel de confianza del $95 \%$ y un margen de error del $7.5 \%$, se obtuvo una muestra total de 168 artesanos, 84 (50\%) correspondientes al estado de Oaxaca, 44 (26.2\%) al estado de Puebla y $40(23.8 \%)$ al estado de Tlaxcala.

Para la recolección de los datos se utilizó un cuestionario estructurado, el cual se administró a través de entrevistas cara a cara. El estudio es exploratorio debido a la escasez de estudios empíricos que analicen temas ambientales en negocios de artesanías.

El desempeño económico se midió en términos de satisfacción del negocio y rentabilidad. Los indicadores utilizados para medir las variables satisfacción del negocio, desempeño ambiental y valores ambientales fueron retomados de la literatura, después de una revisión exhaustiva para identificar aquellos indicadores que mejor se adaptaran al contexto local, atendiendo a las características de los negocios de alfarería. Para medir la rentabilidad se utilizó el rendimiento sobre los activos (ROA), que es la eficiencia con la cual los negocios de alfarería utilizan su base de activos. El sexo y la etnia fueron tratados como variables dummy, con valores de $1=$ masculino y $0=$ femenino, para la etnia 1 = habla lengua indígena y 0 $=$ no habla lengua indígena. Para la variable escolaridad se establecieron tres categorías, 1 = escolaridad básica, $2=$ escolaridad media y $3=$ escolaridad superior. La edad se clasifico en $1=$ artesanos jóvenes, $2=\operatorname{artesanos}$ adultos y $3=$ artesanos maduros.

Con la finalidad de mejorar la validez de los constructos, se realizó una prueba piloto con un número de artesanos igual al 10\% del tamaño de la muestra total (Babbie, 2004). Se pidió retroalimentación de los participantes para asegurar que las preguntas capturaran los conceptos a medir. A cada individuo se le pidió identificar preguntas que no estuvieran claras, que fueran difíciles de responder o sujetas a ciertas tendencias (prejuicios).

Se utilizó análisis factorial, con rotación varimax y normalización de Kaiser, para determinar la consistencia interna de las escalas. Se seleccionaron aquellos reactivos con un factor de carga superior a 0.5. La suma de los ítems validados fue usada para medir cada variable como un solo constructo. Para evaluar la confiabilidad de las escalas se usó el alfa de Cronbach; los valores obtenidos se encuentran dentro del rango de.90 a.94, lo cual indica que el cuestionario tiene una alta confiabilidad (De Faría, 2010). 


\section{Demografía organizacional y variables ambientales}

Las características demográficas de los administradores influyen en diversas acciones del negocio (Tihanyi et al., 2000); no obstante, como lo mencionan Fernández et al., (2006), este tipo de análisis es todavía muy limitado en relación a problemas ambientales. En esta investigación se examina el vínculo entre características demográficas y valores ambientales, y como estas variables influyen en el desempeño económico y ambiental de los negocios de alfarería; se analizan cuatro características socio-demográficas: sexo, escolaridad, etnia y edad. Previas investigaciones han sugerido que la variabilidad en estas características tiene diferentes maneras de relacionarse con los valores ambientales de los individuos (Stern et al., 1993; Vaske et al., 2001).

Por ejemplo, las mujeres son más propensas que los hombres a considerar que la calidad ambiental tiene consecuencias importantes sobre su bienestar personal, el bienestar social y la salud de la biósfera, por lo que muestran valores más biocéntricos; es decir, más centrados en el cuidado de la naturaleza (Stern et al., 1993; Vaske et al., 2001). Similarmente, las personas con un nivel de educación alto muestran también valores más biocéntricos, orientados al cuidado y conservación del ambiente natural (Vaske et al., 2001). Además, la edad y el grupo étnico al que pertenecen las personas influyen en sus valores ambientales; está influencia tiende a ser mayor entre personas jóvenes y entre personas pertenecientes a un grupo étnico. Un cambio de valores puede ser más difícil para per- sonas de edad avanzada (Baas et al., 1993; Egri y Herman, 2000).

Así, sobre la base de investigaciones previas y la teoría sobre demografía organizacional, esta investigación propone una primera hipótesis: que las características demográficas, tales como sexo, escolaridad, edad y etnia, predicen los valores ambientales, los cuales son trascendentales para la toma de decisiones dentro de la organización; es decir, hay una relación significativa entre las características demográficas de los propietarios de negocios de alfarería y sus valores ambientales.

De acuerdo con autores como Khan y Vieito (2013), Kilduff et al., (2000), Miller y Triana (2009), Parrota et al., (2014) y Sobral y Bisseling (2012), la teoría demográfica se ha centrado particularmente en analizar el desempeño como un conjunto de resultados de la empresa, midiendo este desempeño en términos de eficiencia en operaciones, número de innovaciones, rendimiento, razones financieras, desempeño de mercado y satisfacción en el trabajo. Pocos son los estudios que analizan la relación entre valores y resultados de la empresa; así, por ejemplo, Kilduff et al., (2000) plantea que la diversidad cognitiva, vista está como la variabilidad de atributos no observables, tales como actitudes, valores y creencias, tienen un efecto positivo sobre los resultados de la organización. La información del ambiente externo provoca cambios en las cogniciones, las cuales afectan las acciones que ayudan a determinar subsecuentemente el desempeño. Kotter y Heskett (1992) argumentan al respecto que cuando los miembros de un equipo administrativo ven que su empresa está 
logrando el éxito en el mercado llegan a ser incrementalmente más homogéneos en su idiosincrasia y tradiciones. Un mal desempeño, por otro lado, puede crear un sentido de crisis que resulta en una gama más amplia de percepciones y creencias entre los directivos.

Desde la teoría de los mandos superiores, Hambrick y Mason (1984) y Pansiri (2007), señalan que los resultados organizacionales son el reflejo de los valores y base cognitiva de las personas que toman las decisiones dentro de la organización, para quienes los resultados organizacionales, medidos en términos de rentabilidad, crecimiento y supervivencia, son vistos como el producto de una serie de acciones competitivas que pueden ser rápidamente anuladas por los incesantes cambios estratégicos de los oponentes.

Carpenter et al., (2004), retoman el modelo propuesto por Hambrick y Mason (1984) y plantean discrepancias en los resultados de la organización, refiriéndose ya no solo al aspecto económico del desempeño, sino también a aspectos de mercado, sociales y de innovación.

Así, desde la teoría demográfica, así como desde la perspectiva de los mandos superiores de Hambrick y Mason (1984), se han abordado diferentes resultados de la empresa y se observa claramente la evolución del concepto de desempeño; no obstante, la literatura respecto al desempeño ambiental ${ }^{3}$, como variable resultado, sigue siendo muy limitada, aun cuando en la actualidad se ha argumentado arduamente sobre la importancia de la sustentabilidad en las empresas (Ortiz-de-Mandojana et al, 2011). En este sentido, Kassinis y Panayiotou (2006) y Rivera y De León (2005) hacen un primer intento por explicar el desempeño ambiental como resultado de la empresa desde la teoría de los mandos superiores. Kassinis y Panayiotou (2006) estudian la percepción de los directivos (respecto a la influencia de accionistas, instituciones reguladoras, comunidad y empleados) y su relación con el desempeño ambiental de la empresa. De acuerdo con estos autores, las percepciones de los directivos como variables subjetivas pueden tener un efecto positivo sobre el desempeño ambiental.

Rivera y De León (2005) encuentran que el desempeño ambiental se asocia significativamente con la educación de los directivos: las personas con un nivel más alto de educación poseen una habilidad superior para entender problemas complejos e inciertos, tales como aquellos que afectan al ambiente.

Otro tipo de literatura ha indicado la importancia de los valores en el comportamiento ambiental de las personas. Desde esta postura los valores se han analizado a partir de tres orientaciones: valores altruistas, valores biocéntricos y valores egoístas. En la medida en que el ambientalismo se basa en valores altruistas, el interés por el medio ambiente estará estrechamente correlacionado con las preocupaciones y acciones de tipo altruista; por ejemplo, relacionadas con los 
derechos de las minorías, con la pobreza y con programas orientados a la protección del ambiente natural; los valores biocéntricos, por su parte, se refieren al interés por especies no humanas o por la biósfera y; finalmente, los valores egoístas se refieren al auto-interés, quizás la motivación predominante detrás del comportamiento humano (Stern et al., 1993). De acuerdo con la clasificación de valores ambientales, De Groot y Steg (2007) y Helbig (2011) mencionan que los valores egoístas tienen una relación negativa con el interés ambiental, mientras que los valores altruistas y biocéntricos tienen una relación positiva con dicho interés ambiental. Berkhout y Rowlands (2007) encontraron que los valores altruistas mejoran el desempeño ambiental empresarial.

Con base en la literatura previa, es importante probar variables subjetivas, las cuales, frecuentemente, no son probadas de manera formal en hipótesis de investigación. Se reconoce también que existen muchas variables subjetivas importantes, como las creencias y las actitudes, las cuales no son estudiadas en esta investigación. Los valores ambientales pueden ser importantes dentro de la demografía organizacional para explicar los resultados organizacionales. En consecuencia, se propone una segunda hipótesis: los valores ambientales de los artesanos tienen un efecto positivo sobre los resultados empresariales de sus negocios, vistos estos en términos de su desempeño económico y ambiental.

Respecto a los efectos de los valores ambientales en la relación características demográficas -resultados empresariales, desde la teoría demográfica, surge la perspectiva teórica de "procesos inter- vinientes", que propone que las características psicológicas de los individuos son variables que median la relación entre las características demográficas y los resultados empresariales (Lawrence y Brett, 1997; Lawrence, 1997).

En este sentido, la mayoría de los estudios que han examinado el papel de las características psicológicas, o variables subjetivas, intentando explicar la "caja negra" de la demografía organizacional, han analizado "el conflicto" como una variable mediadora que interviene en la relación entre características demográficas y resultados empresariales (De Wit y Greer, 2008: Kearney et al., 2009; Sobral y Bisseling, 2012), otros se han centrado en la comunicación (Smith et al., 1994), y otros más en variables como la reflexión (Schippers et al., 2003).

Por su parte, Kilduff et al., (2000) hacen una aproximación más cercana al análisis de los valores (vistos desde el punto de vista de las cogniciones) al estudiar el efecto de la diversidad demográfica del equipo directivo sobre el desempeño de la empresa; sin embargo, en este estudio se analiza el papel de los valores a través de sus relaciones directas con la diversidad demográfica y el desempeño.

El modelo propuesto en esta investigación rechaza la existencia de una relación directa entre características demográficas y resultados de la empresa. En este modelo, la relación entre características demográficas y resultados está mediada por los valores ambientales. Así, la diversidad en cuanto a características demográficas de los propietarios de negocios de artesanías dará lugar a valores ambientales, los cuales están vinculados a los resultados del negocio, sean económicos y/o am- 
bientales. Por tanto, se propone una tercera hipótesis: los valores ambientales median los efectos de las características demográficas sobre los resultados empresariales de los negocios de artesanías, vistos estos en términos de su desempeño económico y ambiental.

\subsection{Consideraciones del sector artesanal}

En México el sector artesanal se encuentra inmerso dentro de la clasificación de Mipymes, cuya relevancia económica reside en que constituyen el $99.8 \%$ de las empresas. Las organizaciones de este tipo constituyen un factor importante del sistema económico ya que generan el $52 \%$ del PIB y el $72 \%$ de los empleos formales (Secretaría de Economía, 2009). Los negocios de artesanías en su mayoría cuentan con una infraestructura de tipo familiar, donde no se está sujeto a estándares de venta ni de producción, los cambios y mejoras generalmente ocurren de acuerdo a la voluntad e interés de cada artesano. Debido a su limitada capacidad económica, este tipo de empresas suele utilizar una tecnología ineficiente y muchas veces inapropiada, lo cual se combina con una baja capacidad técnica de los artesanos y una carencia de estrategias de negocios elaboradas, lo cual se refleja en la falta de habilidades para comercializar $y$ vender sus productos más eficazmente (Durham y Littrel, 2000; Fillis, 2002).

Cada negocio de artesanías tiene características diferentes, por lo que no existe un método generalizado para competir eficazmente, no tienen control sobre su mercado, porque dependen del turismo; en muchos casos sus ingresos son insuficientes para invertir en innovacio- nes y/o estrategias de producción y comercialización, que le aportan ventajas competitivas (Domínguez et al., 2004).

Las artesanías son elaboradas conforme a antiguos procesos indígenas, el conocimiento sobre su manufactura y su técnica es trasmitido de padres a hijos, fomentándose así las tradiciones y formando parte de la herencia cultural de muchos pueblos (Turok, 1996). Las artesanías se diseñan en talleres que utilizan maquinaria muy simple, sin llegar a formar parte del sector industrial. Son principalmente las manos de los artesanos, su intuición y cosmovisión los elementos que constituyen sus principales recursos, aunado a los insumos materiales que utilizan, que pueden ir desde una piedra hasta cantidades considerables de minerales, madera y agua, principalmente.

A nivel global, en los últimos años, se ha incrementado la preocupación respecto a la preservación del ambiente natural, como consecuencia de los problemas generados por la contaminación provocada por las empresas, como lo argumenta Pulido (2009). El sector artesanal no es ajeno a ello, a pesar de su importancia cultural; la permanencia de las artesanías se ha visto amenazada por el descrédito en el uso de insumos tóxicos y la explotación excesiva de ciertos recursos naturales en su fabricación (Ramírez, 2008). Así, se hace referencia a un tema que vincula la parte cultural con la ecológica, en donde se tendría que pensar en cómo disminuir el impacto ambiental generado por los negocios de artesanías y, a la vez, conservar sus procesos tradicionales que colocan a la artesanía como un símbolo de nuestra cultura e identificación internacional (Sánchez, 2012). 
Tecnología, innovación y estrategia, desde el ámbito ambiental, requieren de fuertes inversiones (Aguilera et al, 2010) y conllevan también, en muchos casos, el cambiar productos y procesos, lo que en el sector artesanal podría representar una mayor originalidad de los productos, abordar conceptos como características demográficas y valores, en un sector tan tradicional y con creencias tan arraigadas a sus costumbres, como es el artesanal, posiblemente lleve a una mejora en sus resultados organizacionales, sin que esto signifique abandonar la forma tradicional de elaborar sus productos.

\subsection{Modelo Hipotético propuesto para los negocios de alfarería}

De acuerdo con la literatura revisada y las características vinculadas al sector artesanal en México, se presenta el siguiente modelo hipotético. Se identifican relaciones entre ciertas características demográficas de los dueños de negocios de alfarería (sexo, escolaridad, etnia y edad) y los resultados empresariales obtenidos por éstos (desempeño económico y ambiental), esta relación está siendo afectada por los valores ambientales de sus propietarios (Diagrama 1).

\section{Relación entre características demográficas y los resultados de la empresa}

Previo al análisis de correlación y regresión lineal, se realizó un análisis factorial con la finalidad de establecer, en función de los reactivos, que los factores integraban cada variable y determinar con ello la consistencia interna de las escalas.

La satisfacción del negocio, vista como la capacidad competitiva de los artesanos en comparación con sus competidores (Wagner y Schaltegger, 2004), presenta una varianza total explicada del $63 \%$ y un alfa de Cronbach de 0.94 .

Los reactivos que componen esta variable se muestran en la Tabla 1.

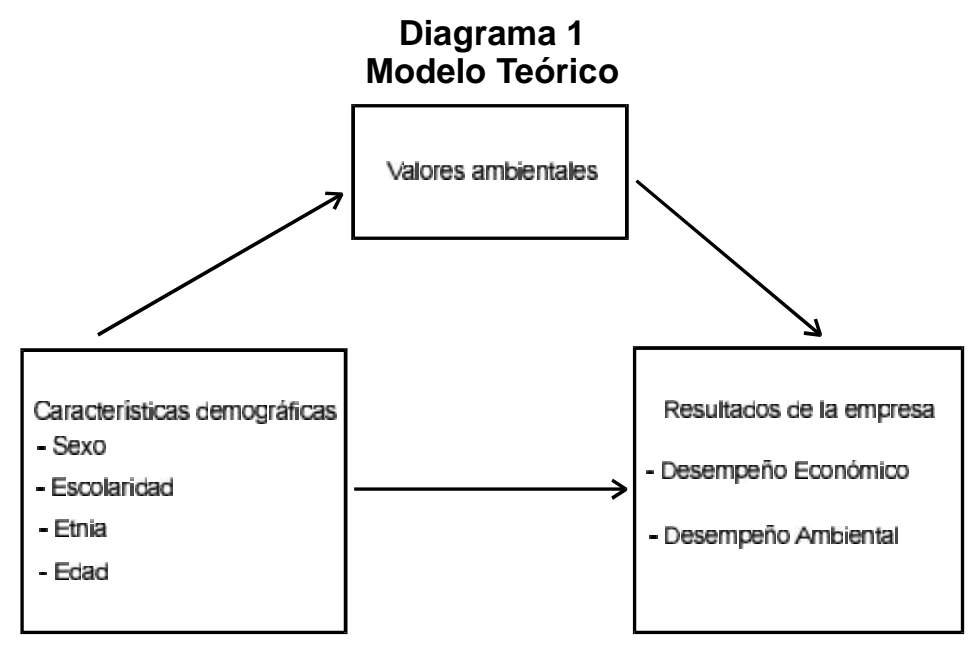

Fuente: Elaboración propia. 


\section{Tabla 1 \\ Análisis factorial de satisfacción del negocio}

\begin{tabular}{|c|c|c|}
\hline $\begin{array}{l}\text { ¿Qué tan satisfecho se siente en cada uno de los siguientes } \\
\text { aspectos, en comparación con su competencia? }\end{array}$ & 1 & Comunalidad \\
\hline \multicolumn{3}{|l|}{ Competitividad interna al negocio } \\
\hline Imagen del producto & .850 & .723 \\
\hline Ventas & .858 & .737 \\
\hline Participación en el mercado & .834 & .696 \\
\hline Nuevas oportunidades en el mercado & .844 & .712 \\
\hline Ventaja competitiva & .829 & .687 \\
\hline Imagen empresarial & .833 & .694 \\
\hline Satisfacción respecto al funcionamiento de su negocio & .682 & .465 \\
\hline Ganancias a corto plazo & .793 & .628 \\
\hline Ganancias a largo plazo & .841 & .707 \\
\hline Ahorro en costos & .739 & .545 \\
\hline Productividad & .739 & .546 \\
\hline Mejores condiciones de trabajo para sus personal & .616 & .379 \\
\hline Varianza explicada & 63 & 63 \\
\hline Alfa de Cronbach & .94 & .94 \\
\hline
\end{tabular}

Método de rotación: Varimax con normalización de Kaiser. La rotación ha convergido en 3 iteraciones. Método de extracción: Análisis de componentes principales.

Fuente: Elaboración propia.

Por otra parte, en lo que respecta al desempeño ambiental, entendido como el resultado ecológico de preservar y proteger el ambiente natural (Sharma 2000; Sharma, 2001; Wagner y Schaltegger, 2004), se integró por 5 cinco factores: residuos tóxicos (factor 1), interés ambiental (factor 2), reutilización (factor 3 ), restitución del daño al ambiente (factor 4 ) y reciclaje (factor 5); esta variable presenta una varianza total explicada del $78 \%$ y un alfa de Cronbach de 0.93. La Tabla 2 muestra los reactivos que componen esta variable, así como las cargas factoriales de cada uno en los factores correspondientes.
Respecto a los valores ambientales, los tres factores resultantes: valores relacionados con la biósfera (factor 1 ), valores altruistas (factor 2 ) y valores egoístas (factor 3 ), explican el $79 \%$ de la varianza, con un alfa de Cronbach de 0.90. La Tabla 3 muestra los reactivos que componen esta variable, así como las cargas factoriales de cada reactivo en los factores correspondientes. Los valores ambientales son entendidos como pautas que enfatizan una relación armoniosa entre el artesano y la naturaleza (Dutcher et al., 2007; Stern et al., 1993). 
Tabla 2

Reactivos que componen la variable desempeño ambiental y sus cargas factoriales

1. Residuos tóxicos

Uso de esmalte sin plomo

.962

Uso de esmalte sin estaño

.963

Uso de esmalte sin cadmio

.963

Uso de esmalte con bajo contenido de plomo

Uso de esmalte con bajo contenido de estaño

Uso de esmalte con bajo contenido de cadmio

Eliminación /tratamiento de sustancias tóxicas

Eliminación o reemplazo de artesanías dañinas

Procesos de producción ambientalmente menos dañinos .733

2. Interés ambiental

Prevención del daño al ambiente

.577

.628

Cambios hechos en el empaque de la artesanía

.871

Uso de empaques biodegradables .819

\section{Reutilización}

Reutilización del barro

Reutilización de minerales

Reutilización del agua

4. Restitución del daño al ambiente

Reforestación

Devolución del jagüete a los yacimientos de donde fue .89 extraído el barro o los minerales

Devolución de otros desechos naturales a los lugares donde .86 fueron extraídos

4

\section{Reciclaje}

Participación en programas de reciclaje

Reciclaje de desechos con otros negocios de

artesanías

Disminución de desperdicios

Varianza explicada

40

18

$.82 \quad .83$
.81

.61

Método de rotación: Varimax con normalización de Kaiser. Método de extracción: Análisis de componentes principales. Fuente: Elaboración propia. 


\section{Tabla 3}

\section{Reactivos que componen la variable valores ambientales y sus cargas} factoriales

¿En qué grado está de acuerdo con cada una de las
siguientes aseveraciones?

\section{Valores relacionados con la biósfera}

Sigue prácticas de conservación del ambiente en su negocio, .81 para evitar la destrucción del ambiente natural

Anima a su personal a adoptar prácticas de conservación del .69

ambiente

$\begin{array}{ll}\text { Participa en actividades del cuidado ambiental para preservar los } & .81\end{array}$

hábitats naturales

Se preocupa por la destrucción del ambiente

Conoce los problemas ambientales que existen en su comunidad $\quad .73$

9

\section{Valores altruistas}

Se Interesa en los problemas ambientales que crea su negocio

de artesanías a su comunidad

$\begin{array}{ll}\text { Evita o sustituye algunas actividades dentro de su negocio que } & .90\end{array}$ podrían perjudicar a sus empleados

Evita el uso excesivo de algunos materiales naturales utilizados

Evita el uso de sustancias tóxicas, por ser dañinas para su salud .80 y la de sus empleados

\section{Valores egoístas}

Evita que otros negocios de artesanías sigan prácticas de conservación al ambiente

Evita que sus empleados adopten prácticas ambientales en sus actividades diarias

Varianza explicada

Alfa de Cronbach

Método de rotación: Varimax con normalización de Kaiser. Método de extracción: Análisis de componentes principales.

Fuente: Elaboración propia. 
La Tabla 4 muestra los estadísticos descriptivos y las correlaciones entre las distintas variables del estudio. Como se observa, hay una relación significativa entre todas ellas, con excepción de la edad y la etnia. riable escolaridad. Se realizaron cuatro modelos de regresión de acuerdo a las características demográficas estudiadas (sexo, escolaridad, etnia, edad) y a las dos variables de resultado (desempeño

\section{Tabla 4}

\section{Matriz de correlaciones entre las variables implicadas}

\begin{tabular}{|c|c|c|c|c|c|c|c|c|c|c|}
\hline & $\mu$ & $\mathrm{s}$ & Sx & E & ES & ED & VA & DE & S & $\mathbf{R}$ \\
\hline Sexo (Sx) & 0.40 & 0.49 & & & & & & & & \\
\hline Etnia (E) & 0.26 & 0.44 & -0.08 & & & & & & & \\
\hline Escolaridad (ES) & 1.86 & 1.26 & $-0.28^{* *}$ & $0.21^{* *}$ & & & & & & \\
\hline Edad (ED) & 1.99 & 0.57 & -0.02 & -0.03 & -0.09 & & & & & \\
\hline $\begin{array}{l}\text { Valores } \\
\text { ambientales (VA) }\end{array}$ & 2.01 & 0.42 & $-0.19^{\star}$ & 0.09 & $0.22^{\star \star}$ & 0.05 & & & & \\
\hline $\begin{array}{l}\text { Desempeño } \\
\text { económico (DE) }\end{array}$ & 3.13 & 0.93 & $-0.21^{* *}$ & 0.06 & $0.38^{\star *}$ & -0.06 & $0.22^{* *}$ & & & \\
\hline Satisfacción (S) & 2.96 & 0.87 & $-0.19^{*}$ & 0.01 & $0.35^{\star *}$ & -0.07 & $0.21^{* *}$ & $0.99^{* *}$ & & \\
\hline Rentabilidad (R) & 0.16 & 0.15 & $-0.24^{\star \star}$ & $0.30^{* *}$ & $0.33^{\star *}$ & 0.06 & 0.14 & $0.47^{\star \star}$ & $0.33^{* *}$ & \\
\hline $\begin{array}{l}\text { Desempeño } \\
\text { ambiental (DA) }\end{array}$ & 2.87 & 0.80 & $-0.15^{\star}$ & $0.33^{\star *}$ & $0.33^{\star \star}$ & -0.11 & $0.29^{* *}$ & $0.39^{\star *}$ & $0.36^{* *}$ & $0.31^{* *}$ \\
\hline
\end{tabular}

$N=168 ;{ }^{* *} p=f 0.01 ;{ }^{*} p=£ 0.05$

Fuente: Elaboración propia.

Las Tablas 5 y 6 muestran los resultados de las regresiones lineales utilizadas para probar las hipótesis de investigación. El factor de inflación de la varianza (FIV) revela que no existen problemas de multicolinealidad entre las variables implicadas, ya que todos los valores están por debajo del valor 5 , como sugieren Aguilera et al. (2010) y Hair et al. (2008). Para el desempeño económico como variable dependiente los valores FIV varían entre 1.02 para la edad y 1.17 para la escolaridad; para el desempeño ambiental como variable dependiente, los valores varían entre 1.01 para la edad y 1.17 para la va- económico y desempeño ambiental).

La primera hipótesis, la cual sugiere una relación significativa entre características demográficas y valores ambientales, fue soportada parcialmente, ya que se encontró una relación significativa entre sexo y escolaridad con valores ambientales $(b=-0.19, p f 0.01$ y $b=0.22, p$ $£ 0.01$, respectivamente), lo cual es consistente con investigaciones previas (Stern et al., 1993; Vaske et al., 2001). Sin embargo, no se encontraron relaciones significativas entre etnia ni edad con valores ambientales $(b=0.09, N S$ y $b=0.05$, $N S$, respectivamente), lo cual contradice 


\section{Tabla 5}

Coeficientes de regresión estimados. Desempeño económico como variable dependiente

\begin{tabular}{|c|c|c|c|c|c|c|}
\hline $\begin{array}{c}\text { Variable } \\
\text { independiente }\end{array}$ & Variable dependiente & $\begin{array}{c}\text { Coeficiente } \\
\text { Beta } \\
\text { estandarizado }\end{array}$ & $\mathbf{t}$ & Sig. & $\mathbf{R}^{2}$ & FIV \\
\hline \multicolumn{7}{|c|}{ Modelo 1 (mediación completa) } \\
\hline Sexo & Desempeño económico & -0.21 & -2.84 & 0.00 & 0.05 & 1.11 \\
\hline Sexo & Valores ambientales & -0.19 & -2.45 & 0.01 & 0.04 & \\
\hline Valores ambientales & Desempeño económico & 0.22 & 2.94 & 0.00 & 0.05 & 1.08 \\
\hline Valores ambientales & Desempeño económico & 0.19 & 2.49 & 0.01 & & \\
\hline Sexo & (regresión múltiple) & -0.18 & -2.37 & 0.20 & & \\
\hline \multicolumn{7}{|c|}{ Modelo 2 (mediación parcial) } \\
\hline Escolaridad & Desempeño económico & 0.38 & 5.26 & 0.00 & 0.14 & 1.17 \\
\hline Escolaridad & Valores ambientales & 0.22 & 2.87 & 0.00 & 0.05 & \\
\hline Valores ambientales & Desempeño económico & 0.22 & 2.94 & 0.00 & 0.05 & \\
\hline Valores ambientales & Desempeño económico & 0.15 & 2.02 & 0.04 & & \\
\hline Escolaridad & (regresión múltiple) & 0.35 & 4.74 & 0.00 & & \\
\hline \multicolumn{7}{|c|}{ Modelo 3 (no hay mediación) } \\
\hline Etnia & Desempeño económico & 0.06 & 0.74 & 0.46 & 0.00 & 1.05 \\
\hline Etnia & Valores ambientales & 0.09 & 1.15 & 0.25 & 0.00 & \\
\hline Valores ambientales & Desempeño económico & 0.22 & 2.94 & 0.00 & 0.05 & \\
\hline Valores ambientales & Desempeño económico & 0.22 & 2.88 & 0.00 & 0.05 & \\
\hline Etnia & (regresión múltiple) & 0.04 & 0.50 & 0.62 & & \\
\hline \multicolumn{7}{|c|}{ Modelo 4 (no hay mediación) } \\
\hline Edad & Desempeño económico & -0.06 & -0.76 & 0.44 & 0.00 & 1.02 \\
\hline Edad & Valores ambientales & 0.05 & 0.66 & 0.51 & 0.00 & \\
\hline Valores ambientales & Desempeño económico & 0.22 & 2.94 & 0.00 & 0.05 & \\
\hline Valores ambientales & Desempeño económico & 0.23 & 2.99 & 0.00 & 0.05 & \\
\hline Edad & (regresión múltiple) & -0.71 & -0.93 & 0.35 & & \\
\hline
\end{tabular}

Fuente: Elaboración propia. 


\section{Tabla 6 \\ Coeficientes de regresión estimados. Desempeño ambiental como variable dependiente}

\begin{tabular}{|c|c|c|c|c|c|c|}
\hline $\begin{array}{c}\text { Variable } \\
\text { independiente }\end{array}$ & Variable dependiente & $\begin{array}{c}\text { Coeficiente } \\
\text { Beta } \\
\text { estandarizado }\end{array}$ & $\mathbf{t}$ & Sig. & $\mathbf{R}^{2}$ & FIV \\
\hline \multicolumn{7}{|c|}{ Modelo 1 (mediación completa) } \\
\hline Sexo & Desempeño ambiental & -0.15 & -2.00 & 0.05 & 0.02 & 1.11 \\
\hline Sexo & Valores ambientales & -0.19 & -2.45 & 0.01 & 0.04 & \\
\hline $\begin{array}{l}\text { Valores } \\
\text { ambientales }\end{array}$ & Desempeño ambiental & 0.29 & 3.90 & 0.00 & 0.08 & 1.08 \\
\hline $\begin{array}{l}\text { Valores } \\
\text { ambientales }\end{array}$ & Desempeño ambiental & 0.29 & 3.90 & 0.00 & 0.08 & \\
\hline Sexo & (regresión múltiple) & -0.10 & -1.36 & 0.17 & & \\
\hline \multicolumn{7}{|c|}{ Modelo 2 (mediación parcial) } \\
\hline Escolaridad & Desempeño ambiental & 0.31 & 4.15 & 0.00 & 0.09 & 1.17 \\
\hline Escolaridad & Valores ambientales & 0.22 & 2.87 & 0.00 & 0.05 & \\
\hline $\begin{array}{l}\text { Valores } \\
\text { ambientales }\end{array}$ & Desempeño ambiental & 0.29 & 3.90 & 0.00 & 0.08 & \\
\hline $\begin{array}{l}\text { Valores } \\
\text { ambientales }\end{array}$ & Desempeño ambiental & 0.23 & 3.12 & 0.00 & 0.16 & \\
\hline Escolaridad & (regresión múltiple) & 0.28 & 3.84 & 0.00 & & \\
\hline \multicolumn{7}{|c|}{ Modelo 3 (no hay mediación) } \\
\hline Etnia & Desempeño ambiental & 0.33 & 4.58 & 0.00 & 0.11 & 1.04 \\
\hline Etnia & Valores ambientales & 0.09 & 1.15 & 0.25 & 0.00 & \\
\hline $\begin{array}{l}\text { Valores } \\
\text { ambientales }\end{array}$ & Desempeño ambiental & 0.29 & 3.90 & 0.00 & 0.08 & \\
\hline $\begin{array}{l}\text { Valores } \\
\text { ambientales }\end{array}$ & Desempeño ambiental & 0.26 & 3.70 & 0.00 & 0.18 & \\
\hline Etnia & (regresión múltiple) & 0.31 & 4.40 & 0.00 & & \\
\hline \multicolumn{7}{|c|}{ Modelo 4 (no hay mediación) } \\
\hline Edad & Desempeño ambiental & -0.11 & -1.41 & 0.16 & 0.01 & 1.01 \\
\hline Edad & Valores ambientales & 0.05 & 0.66 & 0.51 & 0.00 & \\
\hline $\begin{array}{l}\text { Valores } \\
\text { ambientales }\end{array}$ & Desempeño ambiental & 0.29 & 3.90 & 0.00 & 0.08 & \\
\hline $\begin{array}{l}\text { Valores } \\
\text { ambientales }\end{array}$ & Desempeño ambiental & 0.30 & 3.99 & 0.00 & 0.09 & \\
\hline Edad & (regresión múltiple) & -0.12 & -1.67 & 0.09 & & \\
\hline
\end{tabular}

Fuente: Elaboración propia. 
los resultados obtenidos por Baas et al., (1993) y Egri y Herman (2000), pero respaldan lo argumentado por Kilduff et al., (2000), en el sentido de que la demografía tiene efectos sobre la diversidad cognitiva (valores, actitudes y creencias), pero que estos efectos son específicos de ciertos tipos de demografía y ciertos tipos de diversidad cognitiva (véase modelos 1 y 2 de las Tablas 5 y 6 ).

La segunda hipótesis sugiere un efecto positivo de los valores ambientales sobre el desempeño económico y ambiental, vistos como resultados empresariales. Los resultados en las tablas 5 y 6 (modelos 1 a 4) dan soporte a la hipótesis 2: los valores ambientales tienen un efecto positivo sobre el desempeño económi$\mathrm{co}(\mathrm{b}=0.22, p £ 0.05)$ y sobre el desempeño ambiental $(b=0.29, p £ 0.01)$. Este resultado es consistente con la literatura vinculada a la base cognitiva de los actores de poder (Kassinis y Panayiotou, 2006; Kilduff et al., 2000; Pansiri, 2007), aunque no se encontró ningún estudio que reporte la relación entre valores ambientales y desempeño económico reportada en esta investigación.

La tercera hipótesis propone una relación significativa entre las características demográficas y el desempeño económico y ambiental, a través del efecto mediador de los valores ambientales. En la Tabla 5 se presenta al desempeño económico como variable dependiente y se observa que, efectivamente, los valores ambientales median la relación entre sexo y desempeño económico, porque se cumplen las tres condiciones expresadas por Baron y Kenny (1986) para probar mediación: 1) el sexo influye en los valores ambientales $(b=-0.19, p f 0.01) ; 2)$ el sexo influye en el desempeño económico $(b=-0.21, p £ 0.01) y ; 3)$ los valores ambientales influyen en el desempeño económico $(b=0.22, p £ 0.01)$. Finalmente, al ejecutar el análisis de regresión múltiple del sexo y los valores ambientales sobre el desempeño económico, se obtuvo que la relación entre el desempeño económico y el sexo quedo reducida a un coeficiente no significativo $(b=-0.18$, $N S$ ), por tanto se trata de una mediación total (modelo 1, Tabla 5).

En el modelo 2, se observa que los valores ambientales median parcialmente la relación entre escolaridad y desempeño económico porque, efectivamente, se cumplen las tres condiciones anteriores; no obstante, al ejecutar el análisis de regresión múltiple de la variable independiente escolaridad y la variable mediadora valores ambientales sobre la variable dependiente desempeño económico, la relación entre escolaridad y desempeño económico sigue siendo significativa, aunque de menor magnitud (Tabla 5).

En los modelos 3 y 4 no hay efectos de mediación; por tanto, las relaciones etnia - desempeño económico y edad - desempeño económico no están siendo explicadas por la mediación de los valores ambientales.

Respecto al desempeño ambiental como variable dependiente, se tiene en el modelo 1 de la Tabla 6 que hay una mediación total de los valores ambientales en la relación sexo - desempeño ambiental, debido a que (1) el sexo influye significativamente en los valores ambientales $(b=-0.19, p f 0.01)$; (2) el sexo como variable independiente influye significativamente en el desempeño ambiental $(b=$ $-0.15, p f 0.05)$ y; (3) los valores ambien- 
tales influyen en el desempeño ambiental $b=0.29, p £ 0.01$ ). Al efectuar el análisis de regresión múltiple incluyendo la variable independiente sexo y la variable mediadora valores ambientales explicando el desempeño ambiental, se obtuvo que la relación entre desempeño ambiental y sexo quedo reducida a un coeficiente beta no significativo ( $b=-0.10, N S)$.

El modelo 2 de la Tabla 6 muestra una mediación parcial de los valores ambientales en la relación escolaridad y desempeño ambiental. En este caso se cumplen las tres condiciones expresadas con anterioridad; sin embargo, al efectuar el análisis de regresión múltiple, la relación entre escolaridad y desempeño ambiental sigue siendo significativa, aunque en menor magnitud.

Igual que en el caso anterior, en los modelos 3 y 4 no existe mediación de los valores ambientales en las relaciones etnia -desempeño ambiental y edad -desempeño ambiental.

Los resultados hallados de la mediación de la variable valores ambientales en la relación características demográficas -resultados de la empresa apoyan los obtenidos por De Wit y Greer (2008), Sobral y Bisseling (2012) y Schippers et al. (2003), entre otros. No obstante, estos autores analizan el efecto mediador de variables subjetivas, como conflicto, reflexión y comunicación. En la literatura revisada no se localizó ningún artículo que estudiara los valores ambientales como variable subjetiva, como se reporta en este artículo.

\section{Conclusiones}

Una de las corrientes más importantes dentro de la literatura sobre demografía organizacional propone que las características demográficas son indicadores fiables de conceptos subjetivos, que pueden explicar los resultados empresariales; no obstante, en este estudio se concluye que los conceptos subjetivos explican la relación entre características demográficas y resultados de la empresa, como lo plantea la teoría de procesos intervinientes de la demografía organizacional.

La teoría de procesos intervinientes menciona que las características demográficas explican los resultados de la empresa porque ambos, características demográficas y resultados, están relacionados con conceptos subjetivos o intervinientes, concepto que se incorporó en este estudio analizando el efecto mediador de los valores ambientales. Se analizó también el desempeño ambiental como variable resultado; así, en este artículo, se estudia el papel de variables ambientales con la finalidad de contribuir a la teoría sobre demografía organizacional.

Los valores ambientales, conceptualizados como pautas que enfatizan una relación armoniosa entre el artesano y la naturaleza, median la relación entre características demográficas y resultados de la empresa, pero solo en el caso de ciertas características demográficas, como el sexo, donde la mediación es completa, y en la escolaridad, donde la mediación es parcial. La etnia y la edad podrían estar re- 
lacionadas con otro tipo de concepto subjetivo, como lo es el conflicto.

Dada la limitada literatura que existe respecto a los valores como concepto subjetivo de la demografía organizacional, este artículo contribuye a la investigación existente desde la perspectiva de procesos intervinientes, considerando a los valores ambientales como una variable que media la relación entre características demográficas y los resultados de la empresa.

Se demostró empíricamente que el desempeño ambiental es una variable resultado de la empresa y es afectado significativamente por los valores ambientales y por algunas características demográficas como el sexo, escolaridad y etnia, lo cual indica un vínculo entre características demográficas y desempeño ambiental.

Finalmente, hay que señalar algunas limitaciones y futuras líneas de investigación. En primer lugar, ya que en este trabajo de investigación se explica la relación entre características demográficas y los resultados de la empresa a través de una sola característica psicológica, que son los valores ambientales, futuras investigaciones podrían agregar las actitudes o cogniciones con la finalidad de tener una gama más amplia de variables subjetivas y analizar su efecto en la relación entre características demográficas y resultados empresariales. En segundo lugar, sería interesante hacer estudios relacionados con las características demográficas de los artesanos y el medio ambiente en otras ramas artesanales y en otros estados, donde las relaciones pueden variar de acuerdo a la conciencia ambiental generada en cada contexto.

\section{Referencias Bibliográficas}

Aguilera Caracuel; Aragón Correa; Juan Alberto y Hurtado Torres, Nuria Esther (2010). El desempeño medioambiental en empresas multinacionales: influencia de la distancia institucional entre países. Cuadernos de Economía y Dirección de la Empresa, 44, Venezuela, Centro de Estudios de la Empresa Universidad del Zulia, pp 37-58.

Baas, John M.; Ewert, Alan y Chavez, Deborah J. (1993). Influence of ethnicity on recreation and natural environment use patterns: Managing recreation sites for ethnic and racial diversity. Environment Management, 17, 4, United States, Springer-Verlag, pp 523-529.

Babbie, Earl (2004). The practice of social research. $10^{\text {th }}$ Edition. Thomson Wadsworth, United States of America.

Baron, Reuben M. y Kenny, David A. (1986). The moderator-mediator variable distinction in social psychological research: conceptual, strategic, and statistical considerations. Journal of Personality and Social Psychology, 51, 6, United States, American Psychological Association, Inc. pp 1173-1182.

Berkhout, Tom y Rowlands, Ian H. (2007). The voluntary adoption of green electricity by Ontario-based companies: The importance of organizational values and organizational context. Organization Environment, 20, United States, Sage, pp 281-303.

Carpenter, Mason A., Geletkanycz, Marta A. y Sanders, William Gerard. (2004). Upper echelons research revisited: Antecedents, elements, and consequences of top management team composition. Journal of Management 30(6), 749-778. 
CESOP (2012). Las artesanías en México. En Contexto, 20, pp 1-20. México, Centro de Estudios Sociales y de Opinión Pública. Cámara de Diputados.

De Faría, Pirela y Ligia del Carmen (2010). Liderazgo y cultura organizacional en instituciones de educación básica. Revista Venezolana de Gerencia, 15, 51, Venezuela, Centro de Estudios de la Empresa, Universidad del Zulia, pp 486-503.

De Groot, Judith I. M. y Steg, Linda (2007). Value orientations to explain beliefs related to environmental significant behavior: How to measure egoistic, altruistic, and biospheric value orientations. Environment and Behavior, 40, United States, Sage, pp 330-354.

De Wit, Frank R. C., Greer, Lindred L. (2008). The black box deciphered: a meta-analysis of team-diversity, conflict and team performance. Proceedings of the Annual Meeting Academy of Management, Anaheim, CA, USA, 68.

Domínguez Hernández, María Luisa; Hernández, Girón José de la Paz y Toledo, López Arcelia (2004). Competitividad $y$ ambiente en sectores fragmentados. El caso de la artesanía en México, Cuadernos de Administración, 17, 27, Colombia, Redalyc, pp 127158.

Durham, Deborah E. y Littrell, Mary A. (2000). Performance factors of peace corps handcraft enterprises as indicators of income generation and sustainability. Clothing and Textiles Research Journal, 21, United States, Sage, pp 130-141.

Dutcher, Daniel D.; Finley, James C.; Luloff, A.E. y Buttolph Johnson, Janet (2007). Connectivity with nature as a measure of environmental values. Environment and Behaviour, 39, 4, United States, Sage, pp 474-493.
Egri, Carolyn P. y Herman, Susan (2000). Leadership in the North American environmental sector: values, leadership styles, and contexts of environmental leaders and their organizations. Academy of Management Journal, 43, 4, United States, Academy of Management, pp 571-604.

Fernández, Esteban; Junquera, Beatriz y Ordíz, Mónica (2006). Managers' profile in environmental strategy: a review of the literature. Corporate Social Responsibility and Environmental Management, 13, 5, England, WilleyBlackwell, pp 261-274.

Fillis, Ian (2002). Nurturing creative behaviour in the crafts sector. Irish Marketing Review 15, Ireland, Dublin Institute of Technology, pp 38-48.

Haas, Hartmut (2010). How can we explain mixed effects of diversity on team performance? A review with emphasis on context, Equality, Diversity and Inclusion: An International Journal, 29, 5, United Kingdom, Emerald Group Publishing Limited, pp 458490.

Hair, Joseph F.; Anderson, Rolph E.; Tatham, Ronald L. y Black, William C. (2008). Multivariate Analysis, Nueva York, Prentice Hall.

Hambrick, Donald C. y Mason, Phyllis A. (1984). Upper echelons: The organization as a reflection of its top managers. Academy of Management Review, 9, 2, United States, Academy of Management, pp 193-206.

Helbig, Anne Kathrin. (2011). The role of values in environmental behavior. Symposium. The Netherlands, Universtiy of Groningen, pp 1-6.

Jackson, Susan E.; Brett, Joan F.; Sessa, Valerie I.; Cooper, Dawn M.; Johan, Julin A., y Peyronnin, Karl (1991). Some diferences make a difference: individual dissimilarity and group he- 
terogeneity as correlates of recruitment, promotions, and turnover, Journal of Applied Psychology, 76, 2, United States, American Psychological Association, Inc., pp 675-689.

Kassinis, George I., Panayiotou, Aexia (2006). Perceptions matter: CEO perceptions and firm environmental performance (chief executive officers). The Journal of Corporate Citizenship, 23, Brazil, Brazilian School of Public and Business Administration, pp 1-16.

Kearney, Eric; Gerbert, Diether y Voelpel, Sven C. (2009). When and how diversity benefits teams: The importance of team members' need for cognition. Academy of Management Journal, 52, 3, United States, Academy of Management, pp 581-598.

Khan, Walayet A., Vieito, João Paulo (2013). Ceo gender and firm performance. Journal of Economics and Business, 67, Philadelphia, USA, Elsevier, pp 55-66.

Kilduff, Martin, Angelmar, Reinhard y Mehra, Ajay (2000). Top management-team diversity and firm performance: Examining the role of cognitions. Organization Science, 11,1, United States, Informs, pp 21-34.

Kotter, John P. y Heskett James L. (1992). Corporate culture and performance. Free Press, New York.

Lawrence, Barbara S. (1997). The black box of organizational demography. Organization Science, 8, 1, United States, Informs, pp 1-22.

Lawrence, James R. y Brett, Jeanne M. (1997). Mediators, moderators, and test for mediation. Journal of Applied Psychology, 69, 2, United States, American Psychological Association, Inc., pp 307-321.

Miller, Toyah y Triana, María del Carmen (2009). Demographic diversity in the boardroom: Mediators of the board diversity-firm performance relationship.
Journal of Management Studies, 46, 5, England, Wiley-Blackwell, pp 755-786.

Ortiz-de-Mandojana; Natalia, Aragón Correa, J. Alberto y Delgado, Ceballos Javier (2011). La relación entre la propiedad institucional y de los directivos y el desempeño medioambiental. Cuadernos de Economía y Dirección de la Empresa 14 (4), 222-230.

Pansiri, Jaloni (2007). How company and managerial characteristics influence strategic alliance adoption in the travel sector. International Journal of Tourism Research, 9, 4, North America, John Wiley \& Sons Inc., pp 243-255.

Parrotta, Pierpaolo, Pozzoli, Dario y Pytlikova, Mariola (2014). Labor diversity and firm performance, European Economic Review, 66, Philadelphia, USA, Elsevier, pp 144-179.

Pfeffer, Jeffrey (1983). Organizational demography. In L.L. Cummings\& B.M. Staw (Eds.), Research in organizational behavior, págs. 229-357. Greenwich, Conn.: JAI Press.

Pulido, Reinosa Doris (2009). Costos ambientales en el proceso de extracción del aceite de palma. Estudio de un caso. Revista Venezolana de Gerencia, 14, 46, Venezuela, Centro de Estudios de la Empresa, Universidad del Zulia, pp 228-247.

Ramírez, Francisco (2008). Atentan contra artesanía de loza verde. Noticias, México, disponible en: http://www.noticias-oax.com.mx Fecha de consulta: 19.03.2008

Rivera, Jorge y De León, Peter (2005). Chief executive officers and voluntary environmental performance: Costa Rica's certification for sustainable tourism. Policy Sciences, 38, Netherlands, Springer, pp 107-127.

Robb, Alicia M. y Watson, John (2012). Gender differences in firm performance: Evi- 
dence from new ventures in the United States, Journal of Business Venturing, 27, Philadelphia, USA, Elsevier, pp 544-558.

Sánchez, Medina Patricia Soledad (2012). La Administración Ambiental en México: Importancia, reflexiones y prácticas desde el sector artesanal. Editorial Académica Española. Alemania.

Schippers, Michaéla C.; Den Hartog, Deanne N.; Koopman, Paul L. y Wienk, Janique A. (2003). Diversity and team outcomes: the moderating effects of outcome interdependence and group longevity and the mediating effect of reflexivity. Journal of Organizational Behavior, 24, 6, England, WilleyBlackwell, pp 779-802.

Secretaría de Economía. (2009). Observatorio Pyme. Disponible en: http://www.economia.gob.mx/swb/es/econo$\mathrm{mia} / \mathrm{p}$ _cpyme_informacion

Sharma, Sanjay (2000). Managerial Interpretations and Organizational Context as Predictors of Corporate Choice of Environmental Strategy. Academy of Management Journal, 43, 4, United States, Academy of Management, pp. 681-697.

Sharma, Sanjay (2001). Different Strokes: Regulatory Styles and Environmental Strategy in the North-American Oil and Gas Industry. Business Strategy and the Environment, 10, 6, United States, Wiley-Blackwell, pp. 344-364.

Smith, Ken G.; Smith, Ken A.; Olian, Judy D.; Sims, Henry P.; O'Bannon, Douglas P. y Scully, Judith A. (1994). Top management team demography and process: The role of social integration and communication. Administrative Science Quarterly, 39, United States, Sage, pp 412-438.

Sobral, Filipe y Bisseling, Daan (2012). Exploring the black box in brazilian work groups: a study of diversity, conflict and performance. Brazilian Administration Review, 9, Brazil, ANPAD, pp 127-146.

Stern, Paul C.; Dietz, Thomas y Kalof, Linda (1993). Value orientations, gender and environmental concern. Environment and Behavior, 25, 5, United States, Sage, pp 322-348.

Stinchcombe, Arthur L.; McDill Sexton, Mary y Walker, Dollie R. (1968). Demography of organizations. American Journal of Sociology, 74, 3, United States, Universtiy Chicago press, pp 221-229.

Tihanyi, Laszlo; Ellstrand, Alan E.; Daily, Catherine M. y Dalton, Dan R. (2000). Composition of the top management team and firm international diversification. Journal of Management, 26, 6, United States, Sage, pp 1157-1177.

Turok, Martha (1996). Cómo acercarse a la artesanía. Plaza y Valdés (Ed). México, D.F.

Vaske, Jerry J.; Donnelly, Maureen P.; Williams, Daniel R., y Jonker, Sandra (2001). Demographic Influences on Environmental Value Orientations and Normative Beliefs about National Forest Management. Society and Natural Resources, 14, Unied States, Taylor \& Francis Inc., pp 761-776.

Wagner, Marcus y Schaltegger, Stefan (2004). The effect of corporate environmental strategy choice and environmental performance on competitiveness and economic performance: An empirical study of EU manufacturing. European Management Journal, 22(5), 557-572.

Wiersema, Margarethe F., y Bantel, Karen A. (1993). Top management team demography and corporate strategic change. Academy of Management Journal, 35, 1, United States, Academy of Management, pp 91-121. 